\title{
Accuracy of routine digitally guided immediate full-arch rehabilitations: an observational analysis of eight patients
}

\author{
James Kwok Fai Chow ${ }^{1}$, Adam Shui Cheong Siu, Edward Hui ${ }^{2}$, Raymond Lop Keung Chow ${ }^{1}$, \\ Michel Dard ${ }^{3,4}$ \\ 'Department of Oral and Maxillofacial Surgery, Dental Implant and Maxillofacial Centre, ${ }^{2}$ Department of Digital Dentistry, \\ Dental Implant and Maxillofacial Centre, Hong Kong, P. R. China, ${ }^{3}$ Department of Oral Diagnostic and Rehabilitation Sciences, \\ College of Dental Medicine, Columbia University, New York, NY, USA, ${ }^{4}$ Straumann Group, Basel, Switzerland
}

Purpose: Guided surgery might significantly improve the efficacy and predictability of full-arch rehabilitations. This requires highly accurate implant placement. This retrospective statistical analysis of patient records aimed at identifying the factors affecting the implant placement accuracy of guided single and bimaxillary immediate full-arch rehabilitations.

Materials and Methods: Patient, anatomy, procedure, and implant-related parameters that might influence the coronal, apical and angular deviation of the implants and anchoring pins were statistically analyzed using various statistical models: Mixed regression models were used to analyze differences within groups of single parameters individually. Statistically significant associations between placement accuracy and influencing factors after eliminating covariate factors were identified using multivariable mixed regression models.

Results: Patient records of 8 partially edentulous patients treated by four different surgeons and involving 11 procedures, including 61 implants, were analyzed. Average coronal, apical, and angular deviations of $(1.16 \pm 0.80) \mathrm{mm},(1.49 \pm 0.89) \mathrm{mm}$, and $(4.06 \pm 2.17)^{\circ} \mathrm{re}-$ spectively were measured. From the mixed regression analysis, gender, jaw type, implant position, bone resection, and pinhole drill guide were found to have a statistically significant association with one or more accuracy parameters. As part of the multivariable regression analysis after elimination of covariate parameters, the coronal and apical offsets appeared to be influenced by the use of pinhole drill guides $(P<0.001)$ and angular offsets by the surgeon $(P=0.063)$, age $(P=0.027)$, and implant position $(P=0.014)$.

Conclusions: Digital workflows allow for the successful full-arch rehabilitation of partially and fully edentulous patients. The implant placement accuracy might be improved by considering the identified procedure, anatomic, and patient-related factors. (JOURNAL OF DENTAL IMPLANT RESEARCH 2021;40(4):139-150)

Key Words: Digital, Guided surgery, Accuracy, Immediate

\section{INTRODUCTION}

The introduction of digital technologies is rapidly changing the way how dental care is provided today. Digital technologies were first introduced into the dental field for computer-aided design and manufacturing $(\mathrm{CAx})$ of prosthetic parts. Today these technologies are increasingly over spanning and integrating entire treatment workflows in Implantology, prosthodontics, and orthodontics ${ }^{1,2)}$. Computer-guided template-based Implantology (guided surgery) represents a powerful tool to plan and execute implant placement precisely, efficiently, and predictably by using the patients' anatomy and the future prosthetic restoration as a basis for treatment plan-

Received August 13, 2021, Revised September 13, 2021, Accepted September 22, 2021.

(c) Journal of Dental Implant Research.

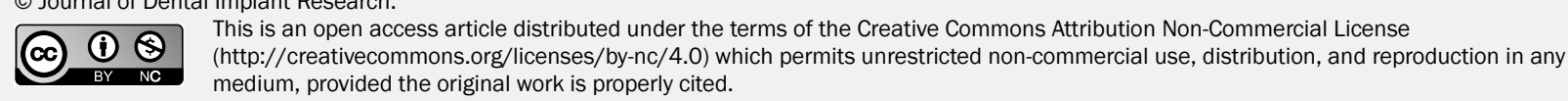

Correspondence to: James Kwok Fai Chow, https://orcid.org/0000-0002-2966-0067

Department of Oral and Maxillofacial Surgery, Dental Implant and Maxillofacial Centre, 1901-05, 12-13, 19/F The Center, 99 Queen's Road Central, Hong Kong, HKSAR, PRC. Tel: +852-2851-4888, Fax: +852-2851-4700, E-mail: jameskfchow@hotmail.com

The authors would like to thank Dr. Albrecht Schnappauf of Dental Wings Inc. for providing technical advice and support regarding coDiagnostiX ${ }^{\mathrm{TM}}$. The authors would like to thank Novonexile AG for writing assistance and editorial support and Leticia Grize for her statistical acumen. 
$\operatorname{ning}^{3,4)}$

Guided surgical approaches involve many individual and success-critical steps that range from collecting and processing 3D dental, osseous, and soft tissue anatomical data to the design and production of surgical guides and prostheses up to implant placement itself ${ }^{5}$. The acquisition of 3D anatomical data is today routinely performed via cone-beam computer tomography (CBCT), intraoral scanners (IOS), desktop scanners (DS), and even face scanners ${ }^{6,7}$. Their main outputs are DICOM data sets (Digital Imaging and Communication in Medicine) and STL files (Standard Triangle Language). These data sets are subsequently integrated into a 3D virtual model of the patient's anatomy, which is used to plan the surgical workflow, to design and manufacture surgical guides and the prosthetic restoration, as well as for the post-processing and verification of the restoration ${ }^{8,9)}$.

Compared to traditional workflows, guided surgical approaches can significantly improve and facilitate the diagnosis, treatment planning, and surgical workflows and render treatments more efficient, predictable, and less time-consuming. These advantages can be of specific importance in advanced indications and complex procedures with challenging or immediate prosthetic objectives like, e.g., immediate full arch reconstructions ${ }^{10-15)}$.

Accurate implant placement and an understanding of the factors and processing steps affecting it are crucial to exploit the full potential of guided surgery ${ }^{16)}$. However, the considerable variability and rapid evolution of applied systems impede a systematic and concluding investigation of such factors, specifically in daily practice settings. Specifically, systematic reviews have not yet shown a significant difference between the accuracy of, e.g., maxillary or mandibular rehabilitations or between fully and partially edentulous treatments. At the same time, individual reports indicate that advanced indications like the rehabilitation of edentulous patients might result in a higher variation and lower implant placement accuracy ${ }^{16,17)}$. This demands further investigations to understand better the factors influencing implant placement accuracy of applied complex guided surgical procedures.

With this descriptive retrospective statistical analysis of selected in-line routine clinical cases, we aim to reinforce the clinical documentation and experience of the factors affecting implant placement accuracy of complex guided surgical interventions. Specifically, we have chosen a systematic multivariate statistical approach to analyze and identify potential effects on multiple levels (patient, anatomical, procedure, and treatment level) on placement accuracy of selected immediate single and bimaxillary full arch restorations.

\section{MATERIALS AND METHODS}

\section{Study design}

This study was conducted as a retrospective descriptive observational statistical analysis of existing patient records of 8 documented clinical cases ${ }^{18)}$. Cases for the analysis were identified and selected by a similar methodology as described by Chmielewski et al. ${ }^{19)}$. Specifically, records of patients that underwent routine immediate full-arch rehabilitations using a guided protocol in a private specialized surgical center in Hong Kong, China, were selected. Selected patient records resulted from the period between May 2017 to May 2018. Procedures were performed by four different surgeons (JC, RC, EH, and $\mathrm{MD}$ ) either alone or in combination (6 different combinations of surgeons). One prosthodontist planned all treatments. Treatment plans were reviewed and approved before treatment in conjunction with the respective treating oral maxillofacial surgeon. Individual cases were selected as part of a review meeting of the authors of this report. Case selection criteria ensured a high consistency in treatment workflows, used restorative materials, and the applied diagnostic tools. Inter-clinician and inter-treatment consistency of the performed treatment workflows were further supported by following the standard operating procedures of the center that were regularly trained as part of joined sessions.

\section{Study population}

In total, records of 8 patients (6 male and 2 female) receiving a total of 11 full-arch rehabilitations were selected. Patients were between 62 and 80 years old at the time of intervention. Treatments comprised 5 maxillary and 6 mandibular arches as part of 2 maxillary, 2 mandibular, and 4 bimaxillary treatments. One maxilla of 
one patient was excluded from the analysis due to the presence of zygomatic implants. All patients had some residual dentition combined with either fixed conventional bridged restorations or removable partial prosthesis. Dental exams at baseline indicated that residual dentitions were affected by advanced caries and/or periodontitis associated with significant alveolar bone loss. The status of residual dentition did not allow for conventional restoration with a good prognosis. 2 patients were under active medication for Osteoporosis, with one patient receiving biannual administration of Denosumab, 4 patients received antihypertensive therapy. None of the patients presented with any local or systemic exclusion criterion for dental implant therapy. All patients signed an informed consent to document, analyze and publish their recorded clinical data.

\section{Diagnostic and virtual treatment planning work- flow}

DICOM data of the patients' osseous and dental anatomy at baseline were obtained from CBCT scans (Newtom 5G, Newtom, Imola, Italy) using a scan prosthesis in a closed bite. Surface STL data of scan prosthesis and soft tissue anatomical contours were generated from impression models using an optical lab scanner (Straumann $^{\circledR}$ CARES $^{\circledR} 7$ series, Institut Straumann AG, Switzerland) ${ }^{15)}$. Scan prostheses of transparent acrylic were prepared using conventional laboratory techniques based on the existing removable partial dentures or temporary dentures. DICOM and STL data sets were registered using radiopaque "fiduciary" markers (Gutta Percha, Dentsply Sirona, USA). Fiduciary markers were incorporated along the rim of the scan prosthesis and under consideration of the dental, hard and soft tissue anatomy in the treatment planning software (coDiagnosti $X^{\mathrm{TM}}$, Dental Wings, Germany). Implant types, positions, abutments, surgical guides, and drill sequences were planned in the treatment planning software (coDiagnosti ${ }^{\mathrm{TM}}$, Dental Wings, Germany) after designing the prosthodontic rehabilitation as digital wax-up (DWOS, Dental Wings, Germany).

Pinhole drill guides (Institut Straumann AG, Switzerland) were tooth or tooth, and mucosa retained depending on the extent of residual dentition. 2 to 4 anchoring pins per arch were used for orientation of bone resection and drilling guides (Guided Surgery Fixation pin, Neodent, Brazil). Mandibular bone resection was performed using bone resection guides (Institut Straumann AG, Switzerland). Serial drill guides equipped with T-Sleeve, $\varnothing 5 \mathrm{~mm}$ (Institut Straumann AG, Switzerland) were used for guided drilling and implant placement.

\section{Implantation and prosthetic rehabilitation}

Patients were instructed for pre-surgical mouth rinse with $0.2 \%$ chlorhexidine for 60 seconds. Surgeries were carried out under monitored anesthesia care (MAC) after local infiltration anesthesia (Xylestesin-A with 2\% Lignocaine (3M ESPE. Guided Straumann ${ }^{\circledR}$ BLT Implants with a diameter of $\varnothing 3.3 \mathrm{~mm}$ narrow collar (NC), Ø 4.1 mm regular collar (RC) or $\varnothing 4.8$ (RC) and with a length between 8 to $14 \mathrm{~mm}$ were placed as instructed by the manufacturer (Institut Straumann AG, Switzerland). Implant placement was carried out after raising an extended mucoperiosteal flap. Drilling was apically and axially controlled with guided drills, and drill handles, respectively, under copious irrigation with sterile saline. Apical depth was controlled by the use of stop keys (Institut Straumann AG, Switzerland). Implant osteotomy fine preparation (contour and thread predrilling) was performed as a function of local bone quality.

Implants were restored with screw-retained abutments (Straumann $^{\circledR}$ Screw-retained Abutment, Institut Straumann, Switzerland, 0 and $30^{\circ}$ angulation, gingival heights $1,2.5$, and $4 \mathrm{~mm}$ ) with a definitive torque of $35 \mathrm{Ncm}$, followed by primary wound closure. Prefabricated prostheses (Institut Straumann, Switzerland) were provisionally installed to verify the correct position of abutments using anchoring pins.

Next non-engaging titanium copings (Institut Straumann AG, Switzerland) were mounted, and the provisional prosthesis was fixed using self-curing acrylic (UniFast Trad (pink), GC America, USA) in a closed bite position. After pickup, implant positions and angulations were registered on a plaster cast model with abutment analogs (Institut Straumann AG, Switzerland). Titanium copings were shortened and polished, anchoring sleeves were removed, and the provisional prosthesis was mounted with a torque of $15 \mathrm{Ncm}$. The temporary provi- 
sional access holes were filled with condensed PTFE tape and temporary composite (Fermit-N, Ivoclar Vivadent, Lichtenstein). Final CBCT scans were acquired for the control of marginal bone levels and implant placement accuracy evaluation.

\section{Evaluation of implant placement accuracy}

Implant placement accuracy was evaluated directly from overlays of pre- and postoperative DICOM data files (treatment evaluation module within coDiagnosti $X^{\mathrm{TM}}$, Dental Wings, Germany) ${ }^{20)}$. Accurate superimposition between scans was achieved by using at least three characteristic and specifically locatable anatomical landmarks per jaw (maxilla: anterior nasal spine, left and right infraorbital processes, margins, and anterior zygomatic bones; mandible: left and right condylar and coronoid processes and mental foramina as well as mental protuberance) (see also Fig. 1). Angular and positional deviations between planned and actual implant positions were derived for all implants and anchoring pins as total distance 3D coronal deviation (deviation at implant platform), 3D apical deviation (deviation at implant tip), or angular deviation of the implant long axis.

\section{Statistical analysis}

Patients' characteristics and clinical parameters were reported as descriptive categorical characteristics and summarized as counts and percentages. Continuous parameters were reported as mean values, standard deviation, and median, including interquartile and total ranges.

The influence of patient, local anatomical, treatment, and material-related factors on the coronal, apical and angular deviation of implants and anchoring pins (outcome) were statistically analyzed using different statistical models.

Patient-related factors included gender and age. Local anatomical factors included jaw type (maxilla, mandible), tooth type (incisor, canine, premolar, molar), quadrant (1
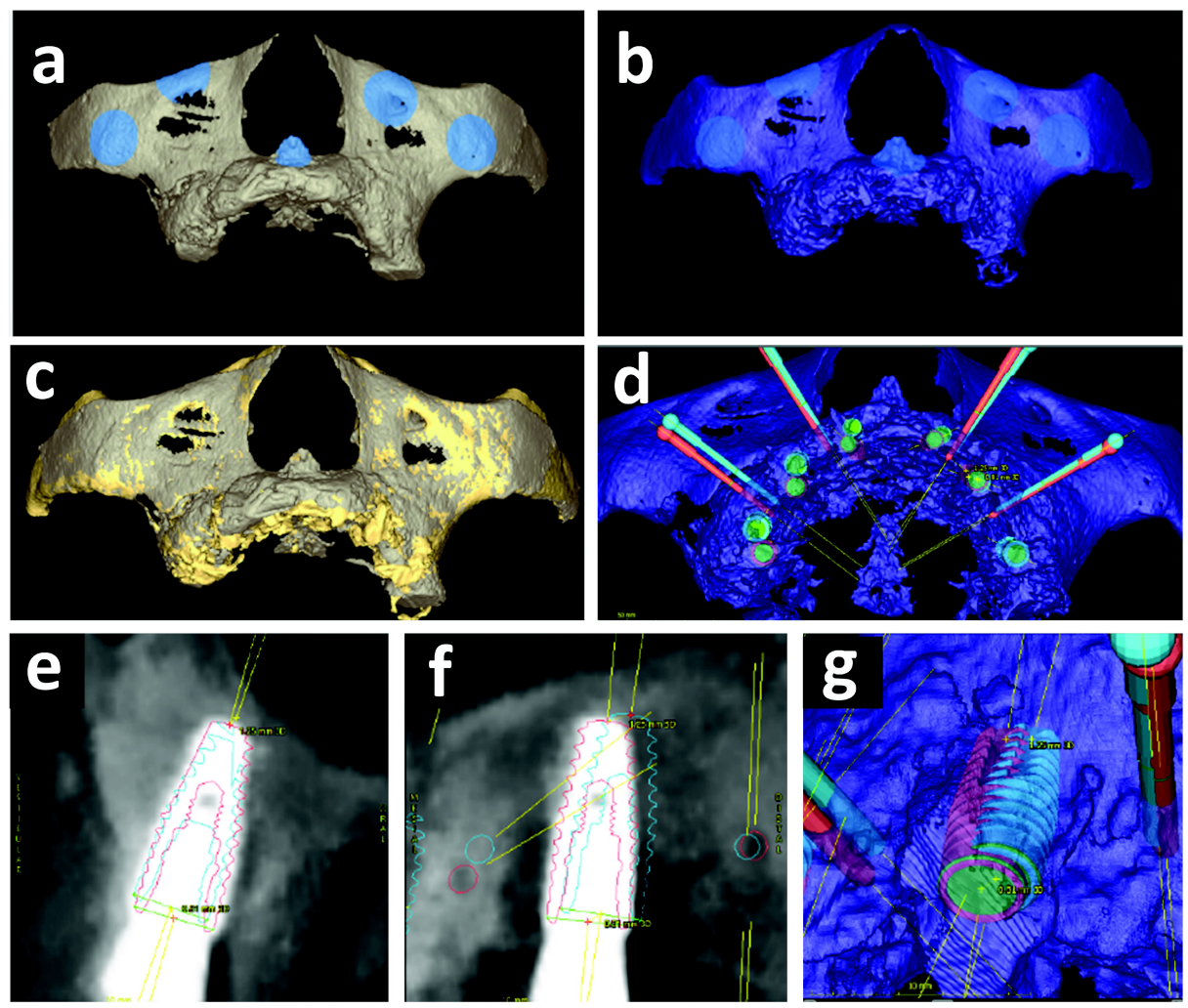

Fig. 1. Alignment pre-and post-surgical CBCT scans and analysis of coronal, apical, and angular offset. (a) and (b) pre-and post-surgical DICOM images. Blue circles designate anatomic landmarks used for alignment. (c, d) matched DICOM images without (c) and with (d) planned and actual anchoring pin and implant positions. (e) and (f) buccolingual and mesiodistal 2D sections of DICOM images. (g) 3D overlay of planned and actual implant positions. Planned and actual positions are shown in blue and red color respectively. 
to 4$)$, position in the quadrant (1 to 7$)$, side of the jaw (left, right).

Treatment-related factors included: anchoring pin hole drill guide use (yes, no), number of anchoring pins $(2,3$, $4)$, drill length (long, extra long), sleeve position ( $\mathrm{H} 2, \mathrm{H} 4$, H6 for 2, 4, and $6 \mathrm{~mm}$ above crest respectively), the operating clinician ( 1 to 6 , coded), bone resection (BR) (yes, no).

Material related factors included: Item or platform type (regular collar (RC), narrow collar (NC), anchoring pin (PIN)), implant diameter $(3.3 \mathrm{~mm}, 4.1 \mathrm{~mm}, 4.5 \mathrm{~mm}, 4.8$ $\mathrm{mm})$, implant length (8 mm, $10 \mathrm{~mm}, 12 \mathrm{~mm}, 14 \mathrm{~mm})$ and the number of implants $(4,6,8)$.

Statistical methods included the following: Method A: Unadjusted comparisons of the outcomes as a function of individual factors were performed by comparing the median of the outcomes at factor level using the non-parametric Kruskal-Wallis test (factors with $>2$ levels) or the Mann-Whitney U-test (factors with 2 levels). Method B: Mixed regression models considering only one factor at the time were used to analyze the association between outcomes and individual factors and compare individual outcomes at the factor level. The patients' effect was taken into account as a random effect in this model. Method C: Multivariable regression models were used to test any statistically significant association between outcomes and influencing factors after eliminating covariate factors. The effect of the patient was included as a random effect. The multivariable models were developed from models considering all factors by the stepwise exclusion of factors with a P-value $>0.2$. For highly correlated factors, the factor resulting in better model performance was retained. Factors with a size effect considered important for the model were precluded from elimination. Model performance was evaluated using the Bayesian information criteria. Values from models B and C were reported as adjusted mean with their $95 \%$ confidence inter- val (CI), and P-values of these models were adjusted for multiple comparisons according to Dunnett-Hsu. The degree of correlation between factors was assessed by calculating Spearman correlations between pairs of factors.

\section{Ethics approval and consent to participate}

Patient records were generated as part of regular and scheduled treatments and with the conscious decision of the patient for the performed treatment after patient information on the treatment alternatives and risks of the treatment. All treated patients signed informed consent for the treatment and the data collection. Ethics approval was not undergone for reviewing and statically analyzing the medical records.

\section{RESULTS}

Patient records considered for the analysis comprised 8 patients ( 2 males and 6 females) with an average age of 69.5 \pm 5.9 years (range: $62 \sim 80$ ) receiving 3 mandibular, 2 maxillary, and 3 bimaxillary full-arch procedures. The analysis of placement accuracy considered a total of 61 implants and 39 anchoring pins that were placed as part of 5 maxillary and 6 mandibular reconstructions comprising 6 to 8 (4 and 1 arch respectively) maxillary implants and 4 to 6 ( 3 arches each) mandibular implants (Table 1, 2). As evidence by the descriptive overview in Table 2 , apical, coronal, and angular offsets between planned and actual positions of implants were consistently and partly statistically significantly higher compared to the corresponding offsets of anchoring pins (apical: $(1.16 \pm 0.80)$ $\mathrm{mm}$ vs. $(0.99 \pm 0.69) \mathrm{mm}, \mathrm{P}=0.4623$; coronal $(1.49 \pm 0.89) \mathrm{mm}$ vs. $(1.019 \pm 0.55) \mathrm{mm}, \mathrm{P}=0.0013$; angular $(4.06 \pm 2.17)^{\circ}$ vs. $\left.(1.70 \pm 2.34)^{\circ}, \mathrm{P}<0.0001\right)$.

The associations between apical, coronal, and angular offsets and individual factors were analyzed using individual mixed regression analysis. The results from this

Table 1. Descriptive patient and procedure-related characteristics

\begin{tabular}{|c|c|c|c|c|c|c|}
\hline Factor & Factor level & $\mathrm{n}$ & $(\%)$ & Average \pm STD & Median (IQR) & Absolute range \\
\hline Age (years) & & 8 & $100 \%$ & $69.5 \pm 5.9$ & $68.5(65.5-73.0)$ & $62 \sim 80$ \\
\hline \multirow[t]{2}{*}{ Age by gender (years) } & Females & 6 & $75 \%$ & $68.3 \pm 4.7$ & $68.5(65-72)$ & $62 \sim 74$ \\
\hline & Males & 2 & $25 \%$ & $73.0 \pm 9.9$ & $73.0(66-80)$ & $66 \sim 88$ \\
\hline
\end{tabular}

$\mathrm{n}$ : Number, STD: Standard deviation, IQR: Interquartile range. 
Table 2. Overview of apical, coronal, and angular offsets stratified by item type and main local anatomical factors

\begin{tabular}{|c|c|c|c|c|c|c|c|}
\hline Factor & Factor level & $\mathrm{n}$ & $(\%)^{*}$ & Average \pm STD & P-value ${ }^{\dagger}$ & Median (IQR) & Absolute range \\
\hline \multicolumn{8}{|c|}{ Coronal offset $(\mathrm{mm})$} \\
\hline \multirow[t]{2}{*}{ Item } & Implants & 61 & NA & $1.16 \pm 0.80$ & 0.4623 & $0.88(0.67-1.54)$ & $0.25 \sim 4.31$ \\
\hline & Pins & 39 & NA & $1.00 \pm 0.69$ & & $0.87(0.53-1.32)$ & $0.07 \sim 2.59$ \\
\hline \multirow[t]{2}{*}{ Jaw type } & Maxilla & 32 & $52 \%$ & $1.40 \pm 0.94$ & 0.0369 & $1.16(0.73-1.74)$ & $0.25 \sim 2.15$ \\
\hline & Mandible & 29 & $48 \%$ & $0.90 \pm 0.50$ & & $0.85(0.57-1.00)$ & $0.48 \sim 4.31$ \\
\hline \multirow[t]{4}{*}{ Tooth position } & Incisor & 21 & $34 \%$ & $1.11 \pm 0.70$ & 0.9762 & $0.97(0.56-1.58)$ & $0.25 \sim 2.83$ \\
\hline & Canine & 1 & $2 \%$ & 1.21 & & $1.21(1.21-1.21)$ & $1.21 \sim 1.21$ \\
\hline & Premolar & 24 & $39 \%$ & $1.12 \pm 0.79$ & & $0.84(0.66-1.51)$ & $0.39 \sim 3.98$ \\
\hline & Molar & 15 & $25 \%$ & $1.29 \pm 0.99$ & & $0.88(0.72-1.54)$ & $0.56 \sim 4.31$ \\
\hline \multicolumn{8}{|l|}{ Apical offset $(\mathrm{mm})$} \\
\hline \multirow[t]{2}{*}{ Item } & Implants & 61 & NA & $1.49 \pm 0.89$ & 0.0013 & $1.33(0.92-1.63)$ & $0.34 \sim 5.20$ \\
\hline & Pins & 39 & NA & $1.02 \pm 0.55$ & & $0.90(0.69-1.21)$ & $0.07 \sim 2.49$ \\
\hline \multirow[t]{2}{*}{ Jaw type } & Maxilla & 32 & $52 \%$ & $1.71 \pm 1.09$ & 0.0666 & $1.44(1.15-1.92)$ & $0.34 \sim 5.20$ \\
\hline & Mandible & 29 & $48 \%$ & $1.24 \pm 0.51$ & & $1.23(0.82-1.47)$ & $0.07 \sim 1.31$ \\
\hline \multirow{4}{*}{ Tooth position } & Incisor & 21 & $34 \%$ & $1.58 \pm 0.75$ & 0.1432 & $1.39(0.62-3.78)$ & $0.62 \sim 3.78$ \\
\hline & Canine & 1 & $2 \%$ & $1.92 \pm 0.00$ & & $1.92(1.92-1.92)$ & $1.92 \sim 1.92$ \\
\hline & Premolar & 24 & $39 \%$ & $1.33 \pm 0.89$ & & $1.24(0.75-1.47)$ & $0.44 \sim 4.28$ \\
\hline & Molar & 15 & $25 \%$ & $1.58 \pm 1.09$ & & $1.41(1.07-1.56)$ & $0.34 \sim 5.20$ \\
\hline \multicolumn{8}{|l|}{ Angular offset $\left({ }^{\circ}\right)$} \\
\hline \multirow[t]{2}{*}{ Item } & Implants & 61 & NA & $4.06 \pm 2.17$ & $<0.0001$ & $3.50(2.60-5.40)$ & $0.60 \sim 9.90$ \\
\hline & Pins & 39 & NA & $1.70 \pm 2.34$ & & $1.40(0.00-2.20)$ & $0.00 \sim 13.00$ \\
\hline \multirow[t]{2}{*}{ Jaw type } & Maxilla & 32 & $52 \%$ & $4.23 \pm 2.44$ & 0.7561 & $3.55(2.45-5.85)$ & $0.60 \sim 9.90$ \\
\hline & Mandible & 29 & $48 \%$ & $3.86 \pm 1.83$ & & $3.50(2.60-4.90)$ & $1.30 \sim 8.80$ \\
\hline \multirow[t]{4}{*}{ Tooth position } & Incisor & 21 & $34 \%$ & $3.75 \pm 2.05$ & & $3.40(2.30-5.40)$ & $0.60 \sim 7.90$ \\
\hline & Canine & 1 & $2 \%$ & 5.50 & & $5.50(5.50-5.50)$ & $5.50 \sim 5.50$ \\
\hline & Premolar & 24 & $39 \%$ & $3.83 \pm 2.05$ & & $3.50(2.45-4.85)$ & $0.90 \sim 8.80$ \\
\hline & Molar & 15 & $25 \%$ & $4.74 \pm 2.50$ & & $4.90(2.60-5.90)$ & $1.60 \sim 9.90$ \\
\hline
\end{tabular}

Factors jaw type and tooth positions report only values related to implants.

n: Number, STD: Standard deviation, IQR: Interquartile range, NA: Not applicable. ${ }^{*}$ Relative percentages using total items per factor as a basis. ${ }^{\dagger}$-value based on Mann-Whitney U-test.

analysis are illustrated and reported in Fig. 2 in the form of the adjusted mean values and their $95 \%$ confidence intervals (CI). Specifically, this analysis evidenced that one or more accuracy outcomes were statistically significantly associated with the factors patient gender, jaw type, implant position, bone resection, and pinhole drill guide. In detail, adjusted average angular offsets in women were smaller when compared to men $\left(3.72^{\circ}\right.$ (CI 3.09 4.34) vs. $5.42^{\circ}$ (CI 4.18 6.65) $(\mathrm{P}=0.017)$. Apical and coronal offsets of mandibular implants were further lower when compared to maxillary implants (apical offset: $1.23 \mathrm{~mm} \mathrm{(CI}$ $0.84 \sim 1.63)$ vs. $1.73 \mathrm{~mm}$ (CI 1.34 2.12), $\mathrm{P}=0.015$; coronal offset: $0.89 \mathrm{~mm}(\mathrm{CI} 0.54 \sim 1.24)$ ) vs. $1.41 \mathrm{~mm}$ (CI 1.05 1.76), $\mathrm{P}=0.038)$. With regards to implant positions, angular offsets showed a statistically significant increase in mesiodistal direction (mesial: $1.78^{\circ}(\mathrm{CI}-0.53 \sim 4.10)$, distal $9.59^{\circ}$ (CI 5.63 to $13.56, \mathrm{P}=0.018$ ). All three accuracy outcomes were consistently lower in procedures involving bone resection compared to procedures without re- section and reached statistical significance for the coronal offset (0.97 mm (CI 0.64 to 1.30 ) vs. $1.43 \mathrm{~mm}$ (CI 1.04 to 1.82), $\mathrm{P}=0.049$ ). Finally, procedures involving a pinhole drill guide showed significantly lower mean apical and coronal offsets compared to procedures that did not use a corresponding guide (apical offset: $1.33 \mathrm{~mm}$ (CI 1.13 $1.54)$ vs. $2.91 \mathrm{~mm}(\mathrm{CI} 2.29 \sim 3.53)(\mathrm{P}<0.001)$; coronal offset: $1.03 \mathrm{~mm}$ (CI $0.84 \sim 1.22)$ vs. $2.36 \mathrm{~mm}$ (CI 1.79 2.93).

Borderline significant associations $(0.01>\mathrm{P}>0.05)$ were further detected between one or more accuracy outcomes and the factors quadrant, number of implants, sleeve position, No of pins and surgeons. Here coronal offsets increased from quadrant $4(0.74 \mathrm{~mm}$ (CI $0.29 \sim 1.18))$ to quadrant 1 (1.43 mm (CI 0.99 1.86) (P=0.070), while implants in reconstructions with 4 implants compared to reconstructions with 6 or 8 implants showed consistently lower apical $(0.87 \mathrm{~mm}$ (CI $0.21 \sim 1.55)$ vs. $1.64 \mathrm{~mm}$ (CI $1.18 \sim 2.10)$ or $1.58 \mathrm{~mm}(\mathrm{CI} 0.66 \sim 2.51)$, $(\mathrm{P}=0.059))$ and coronal offsets respectively $(0.70$ (CI $0.08 \sim 1.30$ vs. $1.25 \mathrm{~mm}$ 


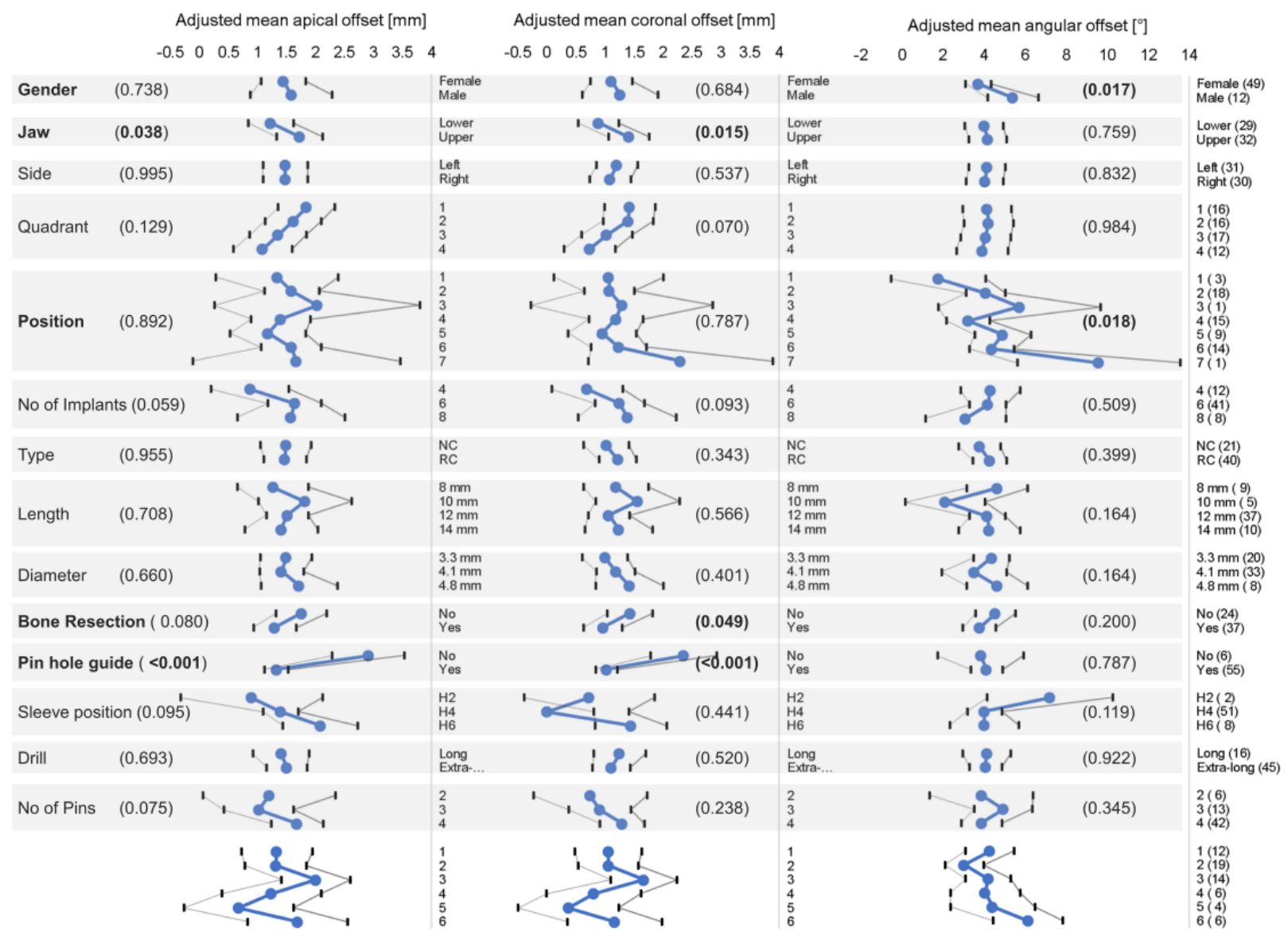

Fig. 2. Adjusted mean apical, coronal, and angular offsets as a function of influencing factors derived from mixed regression models. Values in parenthesis indicate p-values for the overall effect of the corresponding factor. NC: narrow collar, RC: regular collar. Values in parenthesis on the right ordinate designate the sample number at the corresponding factor level group. Factors printed in bold designate factors that show statistically significant $(\mathrm{P} \leq 0.05)$ associations to at least one outcome. Black upper and lower lines demark the upper and lower borders of the $95 \%$ confidence interval.

(CI $0.82 \sim 1.67)$ or $1.38 \mathrm{~mm}(\mathrm{CI} 0.54 \sim 2.23)(\mathrm{P}=0.093)$ ). Further apical offsets appeared lower in implants placed with sleeve heights of $2 \mathrm{~mm}(\mathrm{H} 2)$ compared to 4 or $6 \mathrm{~mm}$ (H4 and H6 respectively) (0.90 $\mathrm{mm}(\mathrm{CI}-0.32 \sim 2.12)$ vs. $1.40 \mathrm{~mm}(\mathrm{CI} 1.10 \sim 1.70)$ and $2.09 \mathrm{~mm}(\mathrm{CI} 1.44 \sim 2.73)$ respectively, $\mathrm{P}=0.095)$. Interestingly apical offsets were further highest when 4 anchoring pins were used $(1.69 \mathrm{~mm}$ (CI 1.24 2.13)) compared to restorations that used $3(1.03$ mm (CI 0.42 1.63)) or 2 pins (1.20 mm (CI 0.06 2.34)) respectively $(\mathrm{P}=0.075)$. Finally, angular deviations were varied between $3.05 \mathrm{~mm}(\mathrm{CI} 2.10 \sim 4.00)$ with surgeon 2 to $6.15 \mathrm{~mm}$ (CI 4.46 7.84) for surgeon $6(\mathrm{P}=0.066)$.

Additional multivariable statistical models were developed and optimized by eliminating covariate factors using backward selection to refine the identification and analysis of influencing factors on the studied offset parameters. The resulting optimized models, including the apical, coronal, and angular adjusted mean offsets as a function of the main influencing factors, are illustrated in Fig. 3. From this analysis, the factors pin hole drill guide $(\mathrm{P}<0.001)$ and jaw type $(\mathrm{P}=0.141)$ were identified to influence the coronal offsets. Apical offsets showed main associations to the factors pinhole guide use ( $P$ $<0.001)$ and sleeve position ( $\mathrm{P}=0.386)$. Adjusted mean angular offsets showed main associations with the factors surgeon ( $\mathrm{P}=0.063)$, age $(\mathrm{P}=0.027)$, and position $(\mathrm{P}=0.014)$. The identified parameters from this model showed similar trends and behaviors as compared to the multivariate models. The factor patient age that was newly identified as an influencing factor as part of this model indicated that the angular offset was reduced by $(0.27 \pm 0.18)^{\circ}$ per year of patient age. 


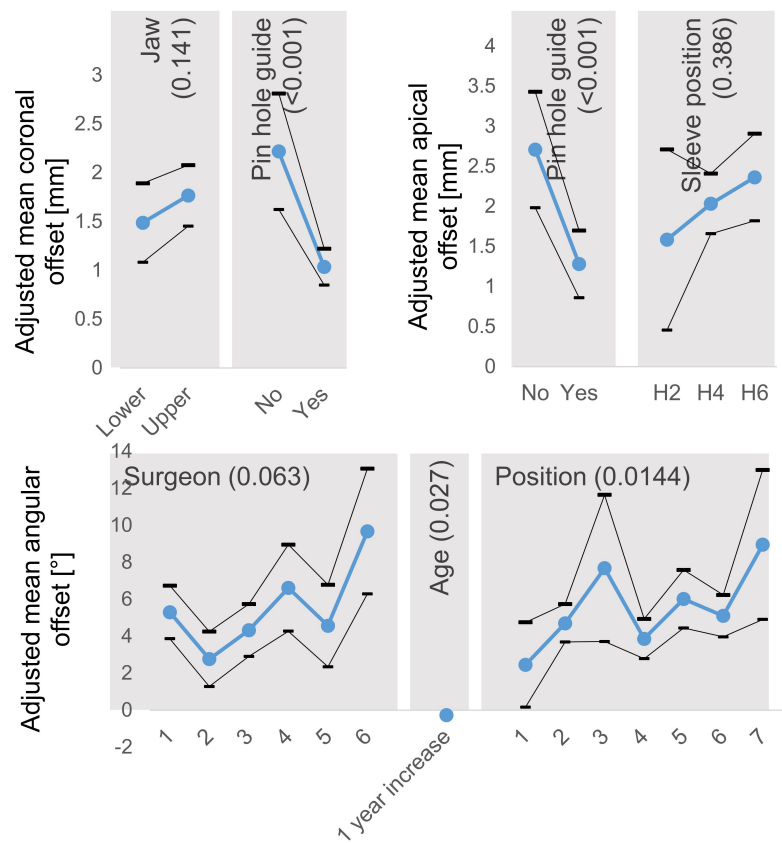

Fig. 3. Adjusted mean apical, coronal, and angular offsets after eliminating covariate factors from multivariable mixed linear regression models. Values in parenthesis indicate P-values for the overall effect of the corresponding factor. Black upper and lower lines demark the upper and lower borders of the 95\% confidence interval. For the factor age, the annual change $(-0.270 \%$ year) is reported.

\section{DISCUSSION}

This retrospective statistical analysis of patient records identified potential factors influencing implant placement accuracy as part of guided immediate full-arch rehabilitation of partially edentulous patients when performed in a routine clinical setting.

Overall implant placement accuracies reported herein were in good agreement with values from the literature. Schneider et al. and Thamaseb et al. have recently published two independent metanalyses of reports addressing implant placement accuracies of guided surgery ${ }^{8,16)}$. Specifically, the authors have reported coronal 3D offsets of $1.16 \mathrm{~mm}$ (range $0.92 \sim 1.39 \mathrm{~mm}$ ) and 1.12 (maximum $4.5 \mathrm{~mm}$ ) respectively, apical 3D offsets of $1.96 \mathrm{~mm}$ (range $1.33 \sim 2.58 \mathrm{~mm}$ ) and 1.39 (maximum $7.1 \mathrm{~mm}$ ) respectively, and angular offsets of $5.731^{\circ}$ (range $3.96 \sim 7.491$ ) and $3.89^{\circ}$ (maximum $21.16^{\circ}$ ) respectively. These values are in good agreement with the accuracy outcomes found within our analysis and well below the proposed safety margins of $2 \mathrm{~mm}$ to critical anatomic structures ${ }^{12)}$. A recent study by
Naziri et al. further indicated that the accuracy of guided implant procedures might be relatively stable over a wide variety of clinical conditions and indications ${ }^{21)}$. This finding may support benchmarking our results to the values from these systematic reviews and the validity of our data set per se.

Raico Gallardo et al. have recently systematically reviewed the available literature in a more differentiated way by considering the influence of tissue support on implant placement accuracy ${ }^{22)}$. With this approach, the author could show that bone-supported guides provided significantly lower accuracies when compared to tooth or mucosa-supported ones. Interestingly, the overall mean apical, coronal, and angular deviations reported herein were consistently lower than the absolute ranges for bone-anchored guides reported by Raico Gallardo et al. and more comparable to those reported for tooth-supported guides. This aspect might be related to the fact that the anchoring pin guides in our study were supported by residual dentition, which may have rendered the positioning of bone-anchored surgical guides and implant placement more accurate.

Both statistical models, i.e., mixed and multivariable ones, identified the following factors to be significantly or partly significantly (significant in one or the other model) associated with one or several implant placement accuracy outcomes: (1) jaw type, (2) pinhole drill guide use, (3) surgeon, (4) position and (5) age of the patient.

The multivariate and the mixed regression models indicated higher offsets in the maxilla than the mandible. Spearman analysis indicated that the factor jaw type was significantly correlated to using a pinhole drill guide, providing higher apical and coronal accuracy. Due to this factor correlation, the observed effect on placement accuracy could not be further resolved and attributed to one individual factor.

Our findings related to the factor jaw type were well in line with conclusions from recent systematic reviews by Alves Marlière et al. and Zhou et al. ${ }^{23,24)}$. Specifically, the authors have identified a predominant tendency for lower implant placement accuracies in the maxilla compared to the mandible throughout the available published literature on guided procedures in partially and fully edentulous patients. To our knowledge, this report is the 
first to confirm this apparent tendency for lower implant-placement accuracies in the maxilla for full-arch restorations of partially edentulous patients.

Interestingly, Ozan et al. have attributed differences in placement accuracy between the maxilla and mandible to alterations in bone density ${ }^{25}$. Variations in bone density and the alveolar ridge dimensions were also identified as risk factors for placement inaccuracies in other studies ${ }^{26}$. In this context, it is interesting to note that our analysis revealed an increased angular placement accuracy with increasing age, which is contrary to the recent review by Zhou et al. ${ }^{24)}$. Chmielewski et al. have recently assessed implant placement accuracy of guided full-arch restorations in fully edentulous subjects in the context of bone densities. Specifically, the authors have assessed bone densities from implant insertion torque and primary implant stability measurements ${ }^{19}$. From their analysis, dense bone was associated with young age, mandibular bone, posterior area, and men as risk factors for placement inaccuracies. Our analysis confirms the findings reported by Chmielewski et al. for full-arch rehabilitation of partially edentulous patients and may potentially also be associated with a decrease of bone density in patients of progressing age.

Bone resection was also identified to significantly increase the coronal placement accuracy as part of the mixed regression models. The factor bone resection was significantly correlated to the parameters pinhole drill guide and jaw type. Recent case reports have reported that sterolithographically produced bone resection guides may improve the bone reduction process's accuracy ${ }^{27-29)}$. These reports did, however, not report on implant placement accuracy. To the best of our knowledge, this is the first report indicating that bone reduction with a surgical guide may improve implant placement accuracy as part of a fully guided procedure.

Also, no clinical studies appear to be available that have systematically investigated the effect of using a pinhole drill guide yet. Despite this lack of available reference data, the observations in this study are well in line with studies that analyzed the effects of the type of guide support on implant placement accuracy ${ }^{16,30)}$. In this context, it is also interesting to note that anchoring pins displayed a relatively higher placement accuracy when com- pared to implants. Therefore, the presented data consistently supports the use of pinhole drill guides for these types of procedures, provided residual dentition is available to provide support. Also, the benefit of pinhole drill guides seems to outweigh the risk of potential errors during their placement, which might propagate into implant placement inaccuracies.

This analysis further indicated a pronounced interoperator dependency. Pearson correlations indicated that the factor surgeon was only significantly correlated to the sleeve height, which only borderline influenced the apical offset outcome. Several studies have addressed the accuracy of template-based guided surgery as a function of the clinician and, specifically, the clinician's experience. Fernandez-Gil et al., e.g., reported significant interoperator differences in angular precision for surgeons of different experience levels ${ }^{31}$. In contrast, Petterson et al. reported substantial differences in apical and angular deviations between surgeons of similar experience ${ }^{32}$. Interestingly Fernandez-Gil et al. attributed the variation of angular precision to differences in the surgical guide design and the distance between the guide and alveolar crest. The fact that Pearson correlations indicated a dependence between the factors surgeon and sleeve height in our study indicate that similar effects might have caused the identified angular offset in our analysis.

Finally, in both statistical models, angular placement accuracy was significantly associated with the tooth position. Pearson correlation indicated that this factor was only related to implant-related factors (implant type, diameter, and length). Putra et al. have recently reported that implant placement is more accurate with bilaterally supported guides when compared to unilaterally supported and distally extended guides ${ }^{30}$. As the used surgical guides were only supported in the anterior regions, placement in the distal areas might have similarly led to higher deflections of the surgical guide and hence higher implant placement inaccuracies. This aspect and the factor sleeve height discussed above may require careful consideration during guide design to optimize implant placement accuracy.

Guided surgical procedures display a relatively high degree of variation regarding indications, methods used for data acquisition and patient virtualization, applied 
surgical workflows, guide-types, and applied systems, all of which may affect implant placement accuracy in a different way $^{26,33)}$.

Specifically, the indication and patient-individual anatomic preconditions have been reported to affect implant placement accuracy significantly. This effect was mainly attributed to differences in the availability of anatomical structures for landmarking during virtualization and the type of tissues that may be used to support the surgical guide $^{30,34)}$. Kholy et al. have recently shown in a bench study setup that the number of teeth used for guide support can have a significant impact on implant placement accuracy ${ }^{35)}$. In the current analysis, the studied patient cohort displayed a relatively high degree of inter-patient variability with regards to the number and position of residual dentition. This high variability rendered correctly quantifying and considering this influencing parameter in the statistical model challenging, impeding its direct consideration. The exclusion of this parameter from the analysis may be regarded as a limitation of the current study. Potential limitations are also associated with this study's pilot character and the relatively low number of patients and procedures that were evaluated. Careful case selection to ensure sample homogeneity, specifically regarding the treated indication, may, on the other hand, have helpt to partly compensate the relatively low number of cases.

Compared to a systematic and prospective analysis of inter- or intraworkflow variations on placement accuracy, this study analyzed the effect of in-line inter-treatment variations within one specific routine standardized and trained fully guided digital workflow applied in the study center. Influencing factors associated with implant placement accuracy were assessed by applying different statistical multivariate models. Few reports in the literature have applied this approach to study implant placement accuracies as part of guided implant placement so far. Matsumura et al. and Chmielewski et al. have recently studied placement accuracy as part of partial restorations and full arch-restorations of fully edentulous patients, respectively ${ }^{19,36}$. The authors commented on specific advantages of a multivariate statistical setup compared to prospective controlled studies. The latter type often mainly analyzes the influence of univariate factors and may be limited in their ability to consider possible confounding and covariate factors ${ }^{36}$. To our knowledge, this is the first study that applies a multivariate statistical approach to the analysis of placement inaccuracies as part of full-arch procedures of partially edentulous patients.

Acquisition and processing of diagnostic data sets and CAD/CAM production of surgical guides of all analyzed treatments were carried out by a similar and comparable procedure, one operator, and without significant variations. Thus, these processing steps were considered constant for the analysis, and placement accuracy was studied as a function of the residual variable patient, anatomical, operator, and procedure-related factors. Interfactor correlations have represented another intrinsic limitation that was related to the chosen study design. This limitation has reduced the ability to resolve and associate accuracy outcomes to one individual factor in the present study. This aspect was considered whenever concluding on specific associations between implant inaccuracies and influencing factors and may potentially be improved by increasing sample numbers.

Under consideration of potential limitations of this study, this report provides some novel findings that may help clinicians design appropriate workflows and guides for the transition of patients with a failing partial residual dentition into a fixed implant-retained full-arch restoration.

\section{CONCLUSION}

This retrospective statistical analysis of in-line routine immediate template-guided full-arch rehabilitations identified the factors jaw type, drill hole guide, implant position, surgeon, and the patients' age as influencing factors. Procedures within the maxilla, without drill hole guides, in posterior positions, and in young patients might be associated with potentially higher risk factors for positional implant placement inaccuracies. Specific alignments and calibration between surgical professionals executing the same surgical guided workflow might help limit treatment to treatment variations and help to limit or overcome the impact of the identified risk factors. Bone resection guides might represent a promising candidate to 
increase placement accuracy, which might be further substantiated as part of future dedicated clinical investigations.

\section{CONFLICT OF INTEREST}

This retrospective analysis was supported on a technical level by Institut Straumann AG, Basel, Switzerland. Prof. M. Dard is an employee of Institut Straumann AG, Basel, Switzerland. None of the authors of this manuscript have any conflict of interest concerning this analysis.

\section{AUTHORS' CONTRIBUTIONS}

Have made substantial contributions to conception and design, or acquisition of data, or analysis and interpretation of data: JKFC, ASCS, EH, RLKC. Been involved in drafting the manuscript or revising it critically for important intellectual content: JKFC, ASCS, MD. Given final approval of the version to be published. Each author should have participated sufficiently in work to take public responsibility for appropriate portions of the content: JKFC, ASCS, MD. Agreed to be accountable for all aspects of the work in ensuring that questions related to the accuracy or integrity of any part of the work are appropriately investigated and resolved: ASCS.

\section{ORCID}

James Kwok Fai Chow, https://orcid.org/0000-0002-2966-0067

Adam Shui Cheong Siu, https://orcid.org/0000-0001-8601-3635

Edward Hui, https://orcid.org/0000-0002-9677-4056

Raymond Lop Keung Chow, https:// orcid.org/0000-0001-5250-8998

Michel Dard, https://orcid.org/0000-0002-9107-996X

\section{REFERENCES}

1. van Noort R. The future of dental devices is digital. Dent Mater 2012;28:3-12.

2. Agarwal WT, Ganz SD, Kugel G. Digital Technologies: A Roundtable Discussion on Changing the Face of Dentistry |
Dentistry Today ; 4.

3. D'haese J, Ackhurst J, Wismeijer D, De Bruyn H, Tahmaseb A. Current state of the art of computer-guided implant surgery. Periodontol 2000 2017;73:121-33.

4. Katsoulis J, Pazera P, Mericske-Stern R. Prosthetically driven, computer-guided implant planning for the edentulous maxilla: a model study. Clinical Implant Dentistry and Related Research 2009;11:238-45.

5. Schubert O, Schweiger J, Stimmelmayr M, Nold E, Güth J-F. Digital implant planning and guided implant surgery - workflow and reliability. Br Dent J 2019;226:101-8.

6. Verstreken K, Van Cleynenbreugel J, Marchal G, Naert I, Suetens $P$, van Steenberghe D. Computer-assisted planning of oral implant surgery: a three-dimensional approach. Int J Oral Maxillofac Implants 1996;11:806-10.

7. Mangano C, Luongo F, Migliario M, Mortellaro C, Mangano FG. Combining intraoral scans, cone beam computed tomography and face scans: the virtual patient. J Craniofac Surg 2018;29:2241-6.

8. Schneider D, Marquardt P, Zwahlen M, Jung RE. A systematic review on the accuracy and the clinical outcome of computer-guided template-based implant dentistry. Clinical Oral Implants Research 2009;20:73-86.

9. Christ A, Heister R, Anderl R. Reverse Engineering for the Utilization of Product Model Data for CAx Workflows in Dental Technology. In: 20th European Concurrent Engineering Conference, ECEC'2014 and the 10th Future Business Technology Conference, FUBUTEC'2014. Bruges, Belgium: EUROSIS-ETI, 2014 [cited 2019 Nov 30] ; 47-54.

10. Bidra AS. Three-Dimensional Esthetic Analysis in Treatment Planning for Implant-Supported Fixed Prosthesis in the Edentulous Maxilla: Review of the Esthetics Literature: THREE-DIMENSIONAL ESTHETIC ANALYSIS. Journal of Esthetic and Restorative Dentistry 2011;23:219-36.

11. Nickenig H-J, Eitner S. Reliability of implant placement after virtual planning of implant positions using cone beam CT data and surgical (guide) templates. Journal of Cranio-Maxillofacial Surgery 2007;35:207-11.

12. Wismeijer D, Joda T, Flügge T, Fokas G, Tahmaseb A, Bechelli D, et al. Group 5 ITI consensus report: digital technologies. Clin Oral Impl Res 2018;29:436-42.

13. Hultin M, Svensson KG, Trulsson M. Clinical advantages of computer-guided implant placement: a systematic review. Clin Oral Implants Res 2012;23:124-35.

14. Colombo M, Mangano C, Mijiritsky E, Krebs M, Hauschild U, Fortin T. Clinical applications and effectiveness of guided implant surgery: a critical review based on randomized controlled trials. BMC Oral Health 2017;17:150.

15. Scherer MD. Presurgical implant-site assessment and restoratively driven digital planning. Dental Clinics of North America 2014;58:561-95.

16. Tahmaseb A, Wismeijer D, Coucke W, Derksen W. Computer technology applications in surgical implant dentistry: a systematic review. Int J Oral Maxillofac Implants 2014;29:25-42.

17. Verhamme LM, Meijer GJ, Boumans T, de Haan AFJ, Bergé SJ, Maal TJJ. A clinically relevant accuracy study of com- 
puter-planned implant placement in the edentulous maxilla using mucosa-supported surgical templates: clinically relevant implant validation of computer-planned implants. Clinical Implant Dentistry and Related Research 2015;17:343-52.

18. Dard M. The Standardised In-Line Clinical Case Series: A New Concept for Real-World Evidence in Dentistry. 2017;3:6.

19. Chmielewski K, Ryncarz W, Yüksel O, Goncalves P, Baek K-W, Cok $S$, et al. Image analysis of immediate full-arch prosthetic rehabilitations guided by a digital workflow: assessment of the discrepancy between planning and execution. Int J Implant Dent 2019;5:26.

20. Pyo S-W, Lim YJ, Koo KT, Lee J. Methods used to assess the 3D accuracy of dental implant positions in computer-guided implant placement: a review. J Clin Med 2019 Jan 7; 8. doi: 10.3390/jcm8010054

21. Naziri E, Schramm A, Wilde F. Accuracy of computer-assisted implant placement with insertion templates. GMS Interdiscip Plast Reconstr Surg DGPW 2016;5:Doc15.

22. Gallardo YNR, da Silva-Olivio IR, Gonzaga L, Sesma N, Martin W. A Systematic Review of Clinical Outcomes on Patients Rehabilitated with Complete-Arch Fixed Implant-Supported Prostheses According to the Time of Loading. J Prosthodont 2019 Aug 21. doi: 10.1111/jopr.13104

23. Alves Marlière D, Demétrio M, Picinini L, De Oliveira R, Chaves Netto HDM. Accuracy of computer-guided surgery for dental implant placement in fully edentulous patients: A systematic review. Eur J Dent 2018;12:153.

24. Zhou W, Liu Z, Song L, Kuo C-L, Shafer DM. Clinical Factors Affecting the Accuracy of Guided Implant Surgery-A Systematic Review and Meta-analysis. J Evid Based Dent Pract 2018;18:28-40.

25. Ozan O, Orhan K, Turkyilmaz I. Correlation Between Bone Density and Angular Deviation of Implants Placed Using CT-Generated Surgical Guides: Journal of Craniofacial Surgery 2011;22:1755-61.

26. Putra RH, Yoda N, likubo M, Kataoka Y, Yamauchi K, Koyama S, Cooray U, Astuti ER, Takahashi T, Sasaki K. Influence of bone condition on implant placement accuracy with computer-guided surgery. Int J Implant Dent 2020;6:62.

27. Beretta M, Poli P, Tansella S, Maiorana C. Virtually guided alveo- lar ridge reduction combined with computer-aided implant placement for a bimaxillary implant-supported rehabilitation: A clinical report. The Journal of prosthetic dentistry 2018 Feb 8; 120. doi: 10.1016/j.prosdent.2017.11.010

28. Faeghi Nejad M, Proussaefs P, Lozada J. Combining guided alveolar ridge reduction and guided implant placement for all-on-4 surgery: A clinical report. The Journal of Prosthetic Dentistry 2016;115:662-7.

29. Mijiritsky E, Ben Zaken H, Shacham M, Cinar IC, Tore C, Nagy $\mathrm{K}$, et al. Variety of surgical guides and protocols for bone reduction prior to implant placement: a narrative review. IJERPH 2021;18:2341.

30. Putra RH, Yoda N, Astuti ER, Sasaki K. The accuracy of implant placement with computer-guided surgery in partially edentulous patients and possible influencing factors: A systematic review and meta-analysis. J Prosthodont Res 2021. doi: 10.2186/jpr.JPR_D_20_00184

31. Fernández-Gil Á, Gil H, Velasco M, Vázquez J. An In Vitro Model to Evaluate the Accuracy of Guided Implant Placement Based on the Surgeon's Experience. Int J Oral Maxillofac Implants 2017;32:515-24.

32. Pettersson A, Kero T, Söderberg R, Näsström K. Accuracy of virtually planned and CAD/CAM-guided implant surgery on plastic models. J Prosthet Dent 2014;112:1472-8.

33. Kernen F, Kramer J, Wanner L, Wismeijer D, Nelson K, Flügge T. A review of virtual planning software for guided implant surgery - data import and visualization, drill guide design and manufacturing. BMC Oral Health 2020;20:251.

34. Wulfman C, Naveau A, Rignon-Bret C. Digital scanning for complete-arch implant-supported restorations: A systematic review. The Journal of Prosthetic Dentistry 2020;124:161-7.

35. El Kholy K, Lazarin R, Janner SFM, Faerber K, Buser R, Buser $D$. Influence of surgical guide support and implant site location on accuracy of static Computer-Assisted Implant Surgery. Clin Oral Impl Res 2019;30:1067-75.

36. Matsumura A, Nakano T, Ono S, Kaminaka A, Yatani H, Kabata D. Multivariate analysis of causal factors influencing accuracy of guided implant surgery for partial edentulism: a retrospective clinical study. Int J Implant Dent 2021;7:28. 